\title{
Archéopages
}

Archéopages

Archéologie et société

40 | 04-07/2014

Villages

\section{Archéologie d'un village du Moyen Âge à la période moderne. L'exemple de Villiers-le-Bel}

\section{François Gentili}

\section{(2) OpenEdition}

1 Journals

Édition électronique

URL : https://journals.openedition.org/archeopages/639

DOI : 10.4000/archeopages.639

ISSN : 2269-9872

\section{Éditeur}

INRAP - Institut national de recherches archéologiques préventives

\section{Édition imprimée}

Date de publication : 1 juin 2015

Pagination : 132-139

ISSN : 1622-8545

\section{Référence électronique}

François Gentili, « Archéologie d'un village du Moyen Âge à la période moderne. L'exemple de Villiersle-Bel », Archéopages [En ligne], 40 | 04-07/2014, mis en ligne le 01 juillet 2016, consulté le 21 janvier 2022. URL : http://journals.openedition.org/archeopages/639; DOI : https://doi.org/10.4000/ archeopages. 639 


\section{Archéologie d'un village du Moyen Âge à la période moderne L'exemple de Villiers-le-Bel}

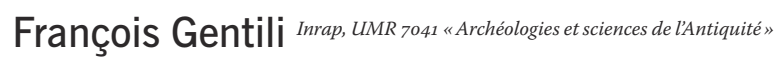

132

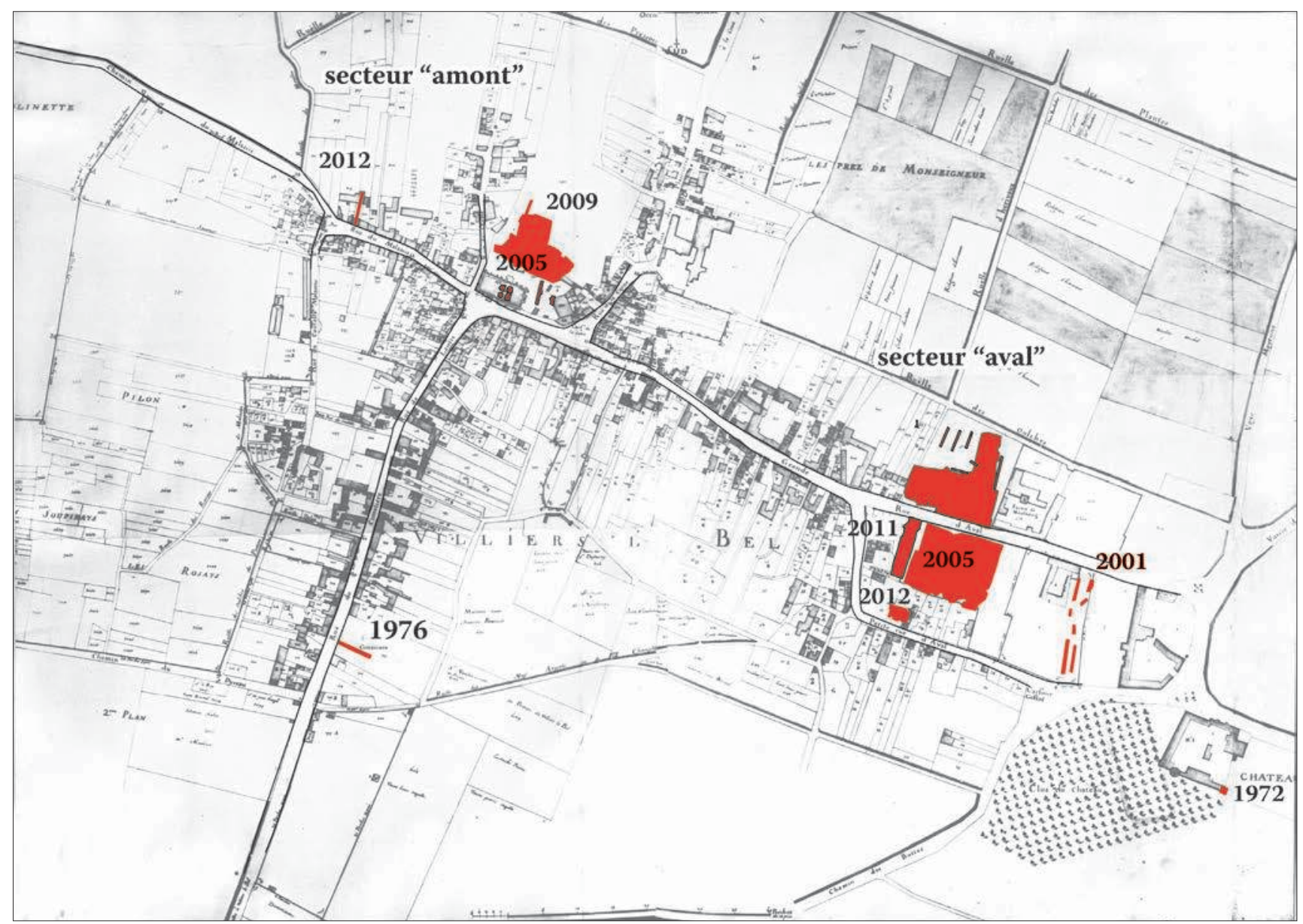


Le développement de l'archéologie préventive a permis depuis plusieurs décennies de mieux comprendre l'émergence de grands habitats groupés durant le Moyen Âge. La fouille de sites complexes associe parfois ensembles funéraires, lieu de culte, habitat privilégié jusqu'à former de véritables agglomérations rurales dépassant la dizaine d'hectares insérés dans un terroir exploité par un maillage d'habitats plus modestes. Néanmoins, la plupart des sites étudiés sur de grandes superficies, notamment en Île-de-France, sont abandonnés entre le $\mathrm{x}^{\mathrm{e}}$ et le $\mathrm{xI}^{\mathrm{e}}$ siècles et ne permettent pas d'étudier l'évolution des formes villageoises durant les siècles qui suivent. Cette question ne saurait être traitée sans avoir recours à l'exploration du sous-sol des villages actuels. Elle nous permet également d'appréhender l'évolution de ces habitats et de leur culture matérielle au Moyen Âge, période souvent absente des habitats fouillés dans les champs (Gentili, 2011)

Au nord de Paris, plusieurs communes présentent des cœurs de villages anciens où dominent des constructions rurales utilisant massivement le plâtre du fait de la proximité des ressources en gypse. La dégradation de ce bâti ancien faute d'entretien et la pression immobilière conduisent souvent à des chantiers de reconstruction touchant le bâti ancien et ses jardins sur des surfaces significatives qui commencent à donner des résultats. Depuis une quinzaine d'années, des bourgs comme Roissy-en-France, Tremblay-en-France, Sarcelles, Villiers-le-Bel, Louvres (Île-de-France) fournissent une image foisonnante et complexe des habitats qui précèdent une " pétrification » (mise en place d'un habitat en dur plus resserré) souvent tardive du village.

À Villiers-le-Bel (Val-d'Oise), six opérations menées depuis 2004 illustrent bien le potentiel de cette archéologie villageoise, mais aussi sa complexité (Gentili, 2012). Cette commune située à une vingtaine de kilomètres au nord de Paris, au cœur de l'ancien Pays de France, est implantée sur le flanc oriental de la butte témoin d'Écouen où la présence de bancs de gypse a favorisé de longue date la fabrication du plâtre et son utilisation dans l'architecture rurale de cette zone. Deux secteurs occupés dès la période carolingienne et distants de $500 \mathrm{~m}$, dans le secteur aval et autour de l'église, permettent d'observer l'évolution du village et de ses différentes composantes sur une longue durée.

\section{La stabilité et la continuité de la période carolingienne à la fin du XII ${ }^{\mathrm{e}}$ siècle}

Trois fouilles contiguës (2004, 2005 et 2011) et un diagnostic (2012) ont été effectués en partie aval du village, sur une superficie avoisinant 1 ha (parcelle de la Confiserie) située de part et d'autre de l'ancienne rue d'Aval. On y observe l'implantation, dès la fin $\mathrm{du}_{\mathrm{VIII}} \mathrm{e}^{\mathrm{s}}$ siècle, d'un habitat très dense sur poteaux plantés, accompagné de fonds de cabanes, fours et silos.
Cet habitat s'installe en bordure de voirie et se développe également plus en profondeur, organisé au sein d'un enclos fossoyé. Le point le plus remarquable est la stabilité de cette occupation depuis la période carolingienne jusquà la fin du XII ${ }^{\mathrm{e}}$ siècle. On observe parfois une superposition des bâtiments de même fonction, greniers, habitations, cabanes avec fosses d'ancrage de métiers et silos; seuls les fours culinaires disparaissent des dernières phases ( $\mathrm{XI}^{\mathrm{e}}-\mathrm{XII}^{\mathrm{e}}$ siècles). Le plâtre est utilisé pour la réalisation des murs à partir du $\mathrm{IX}^{\mathrm{e}}$ siècle, mais son emploi se généralise au XI ${ }^{\mathrm{e}}$ siècle, où il se substitue simplement au torchis sur les murs en clayonnage. Sur le site de Villiersle-Sec, voisin d'une dizaine de kilomètres, la présence d'un bâtiment carolingien aux murs plâtrés constituait un indice social. À Villiers-le-Bel, près de la ressource, il semble qu'il se soit démocratisé.

\section{L'habitat du second Moyen Âge}

La mutation majeure se produit au XIII ${ }^{\mathrm{e}}$ siècle. Il s'agit de l'apparition, sur une surface plus restreinte en bordure de voie, de constructions avec fondations de pierres liées au plâtre. En même temps que le bâti sur poteau planté disparaissent également silos et fonds de cabanes avec fosses d'ancrage, les seules annexes excavées étant des cabanes de formes allongées peut-être liées à la stabulation, et un type récurrent: les caves à cellules latérales.

L'habitat en dur qui fait suite à l'occupation des $\mathrm{VIII}^{\mathrm{e}}-\mathrm{XII}^{\mathrm{e}}$ siècles reprend la trame parcellaire précédente qui était délimitée par des fossés et l'est maintenant par des murs. Une maison-bloc, construite sur un cellier semi-excavé, présente au moins un étage et un comble. Il donne sur une cour dans laquelle se trouve une cave entièrement creusée dans le substrat limoneux et maçonnée avec les murs en plâtre. Un autre habitat en dur ( $\mathrm{XIII}^{\mathrm{e}}$-XIV ${ }^{\mathrm{e}}$ siècles) a été repéré en partie nord, plus à l'ouest, sur la parcelle voisine. Il est également associé à une cave à cellules latérales. La poursuite des fouilles sur les parcelles voisines en 2010-2012 (70, rue Gambetta) nous montre également le développement important de l'habitat médiéval ( $\mathrm{XIII}^{\mathrm{e}}-\mathrm{XV}^{\mathrm{e}}$ siècles) et nous apporte d'autres exemples de ces caves à cellules latérales, dont l'architecture modulaire, va du plus simple - couloir avec escalier à peine maçonné - à un ensemble plus imposant, formé de cinq cellules échelonnées sur une vingtaine de mètres de long. Ces caves sont liées à l'activité vitivinicole très présente à Villiers-le-Bel, mais ont pu également permettre la conservation d'autres denrées.

Enfin, la périphérie de ces habitats livre encore des constructions sur poteaux de taille modeste, mais qui montrent une survivance de ces techniques de construction jusqu'au XIV ${ }^{\mathrm{e}}$ siècle. La mention voisine d'une rue où étaient les loges au $\mathrm{Xv}^{\mathrm{e}}$ siècle nous fournit peut-être un nom pour ces constructions légères (Renaux, 1981). 

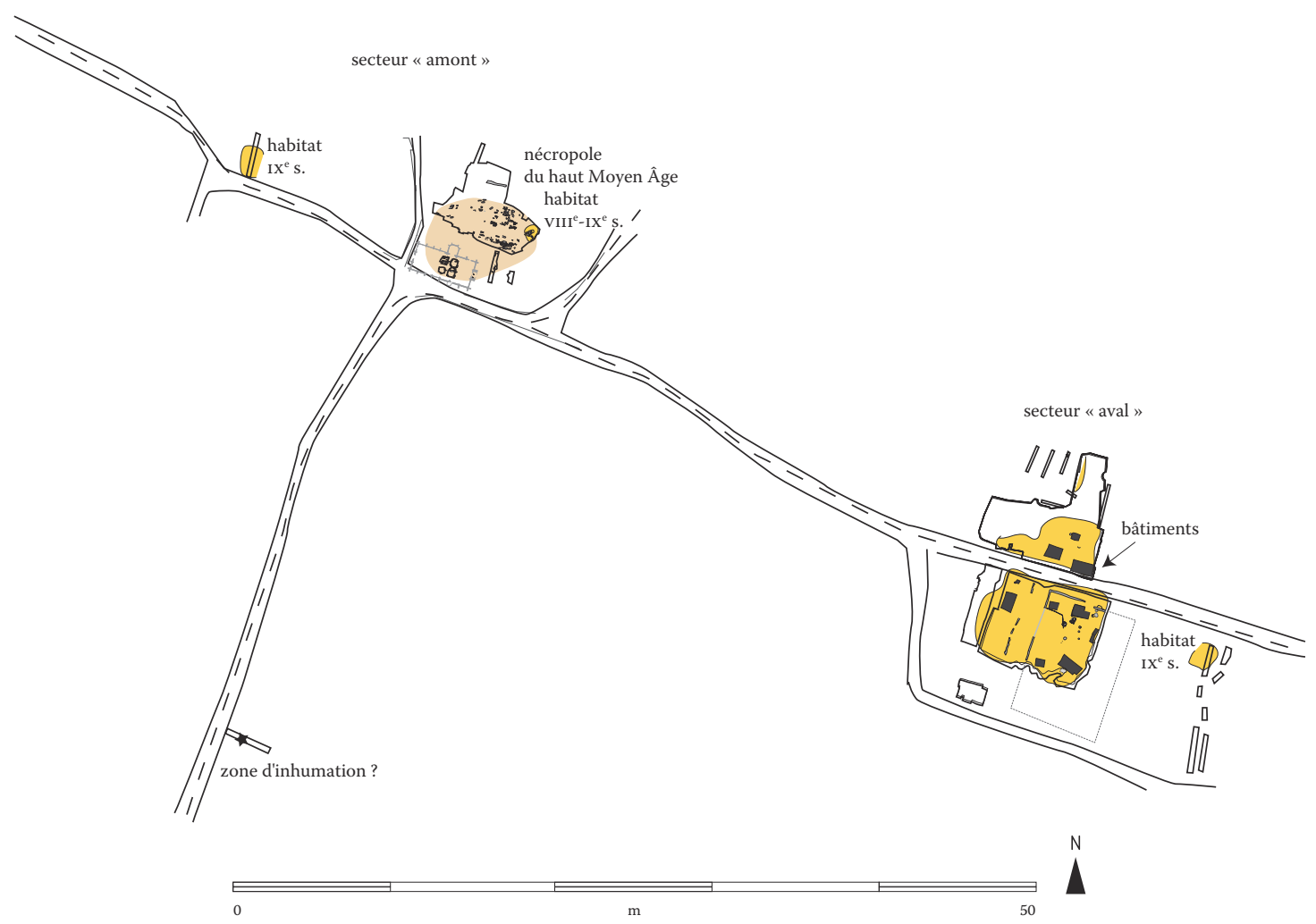

L'ouf, la poule... et le coq : pôles funéraire, cultuel et seigneurial au cour du village

Cette évolution très lente de l'habitat, avec une stabilité évidente du VIII ${ }^{\mathrm{e}}$ au XII ${ }^{\mathrm{e}}$ siècle et une pétrification tardive et progressive, est une caractéristique notoire de la partie aval du village. La permanence de la structure sociale et celle de la propriété du sol peuvent expliquer cette continuité dans les formes et l'implantation de l'habitat. Malgré les changements dans les techniques de construction et la culture matérielle, on observe même des traits de continuité entre l'habitat en dur des $\mathrm{XIII}^{\mathrm{e}}-\mathrm{XV}^{\mathrm{e}}$ siècles et les périodes qui précèdent.

Le contraste est saisissant si on compare avec l'îlot de l'église (parcelle Saint-Didier), où viennent s'entrechoquer, dans des mutations parfois drastiques, habitat paysan, espace funéraire, habitat seigneurial et bâtiments religieux. En effet, deux fouilles, l'une effectuée en 2005 dans l'église actuelle à l'occasion de la restauration du clocher (Gentili, 2008b), l'autre en 2009 sur une large parcelle située au nord de l'église à l'occasion de la reconstruction du collège Saint-Didier ( 0,4 ha), nous apportent une vaste fenêtre de lecture dans un secteur clé qui regroupe les différentes composantes du village.

Les enjeux ne sont pas mineurs puisqu'il s'agit de comprendre comment s'est forgé ce cœur de village et le rôle respectif du cimetière, de l'église et du « château » dans cette émergence d'un lieu maintenant largement dominé par une vaste église gothique et Renaissance. La présence de vestiges antiques sous les villages actuels a longtemps été avancée pour expliquer leur origine, le scénario proposé étant le regroupement de la population des habitats dispersés environnants autour d'un édifice cultuel, lequel aurait attiré ensuite le cimetière (Guadagnin, 1982). Cette explication, donnée notamment à Villiers-le-Bel (Guadagnin, 1981), a souvent été reprise, avec en corollaire l'idée d'une christianisation précoce, « dès la fin de l'Antiquité» (Foussard, 2008, p. 289). 

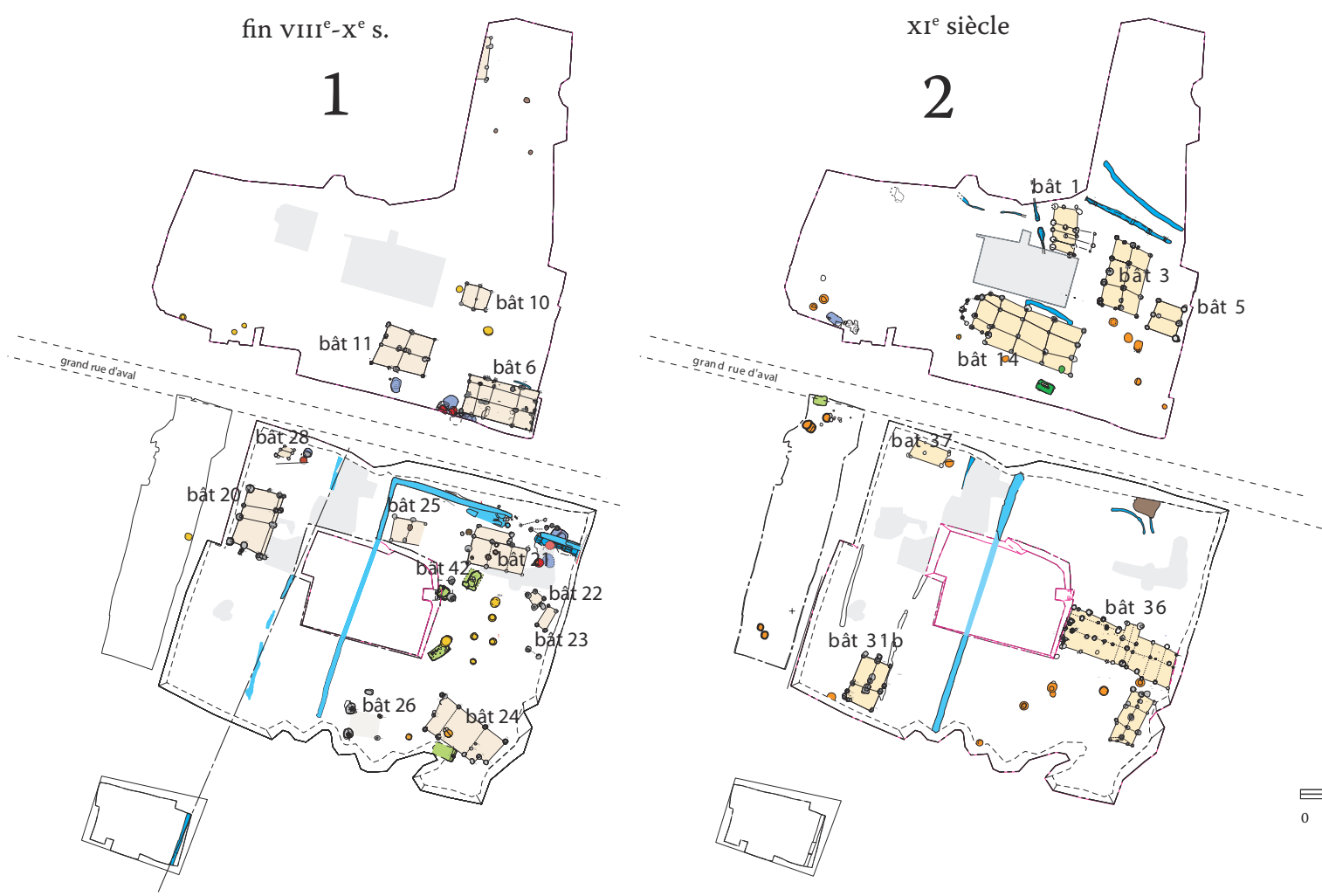

Fond de cabanes Cendrier

Four culinaire

Fossé

Fossé ind.

L Mur

- Silo à grain

Zone détruite

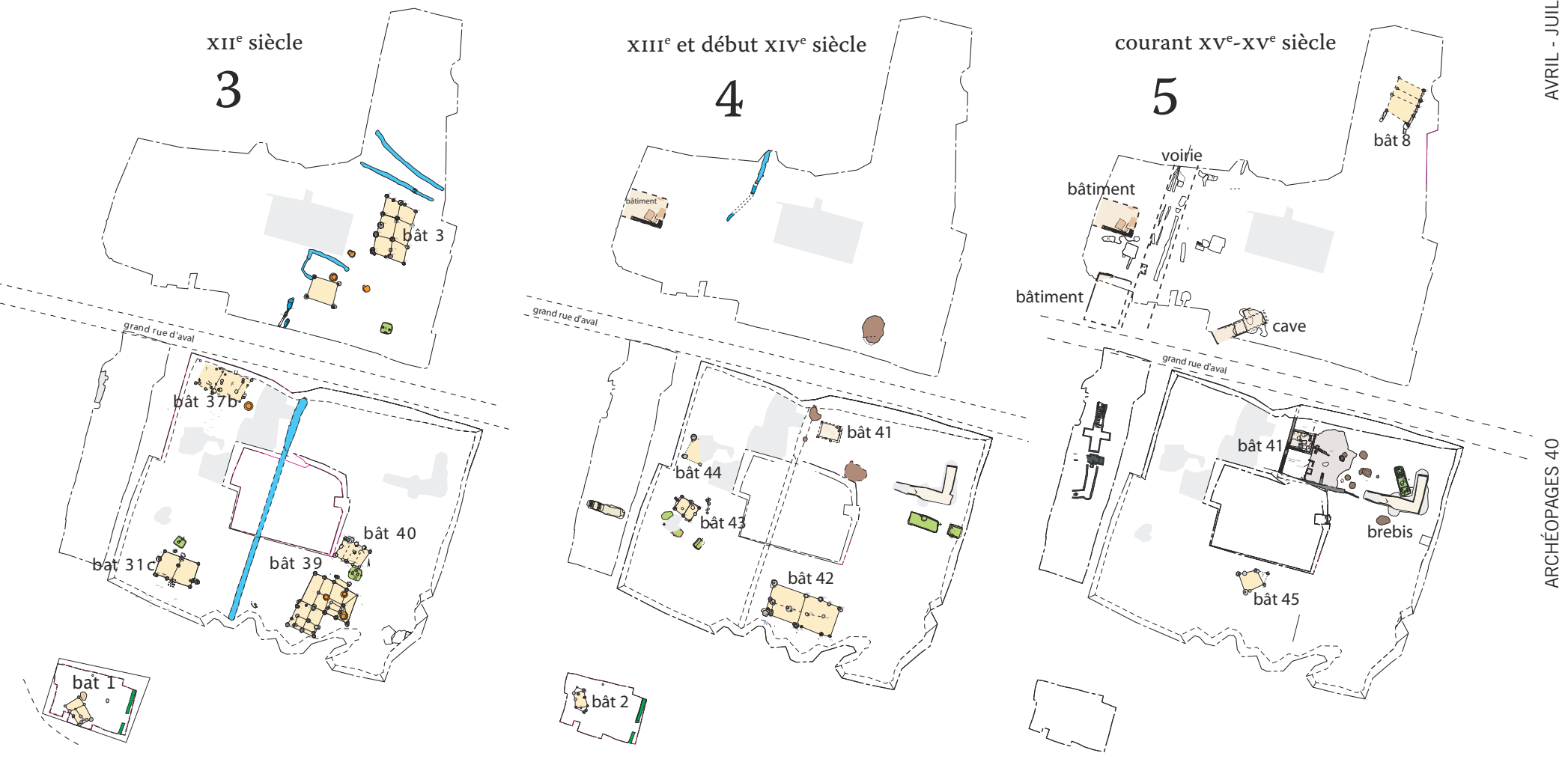



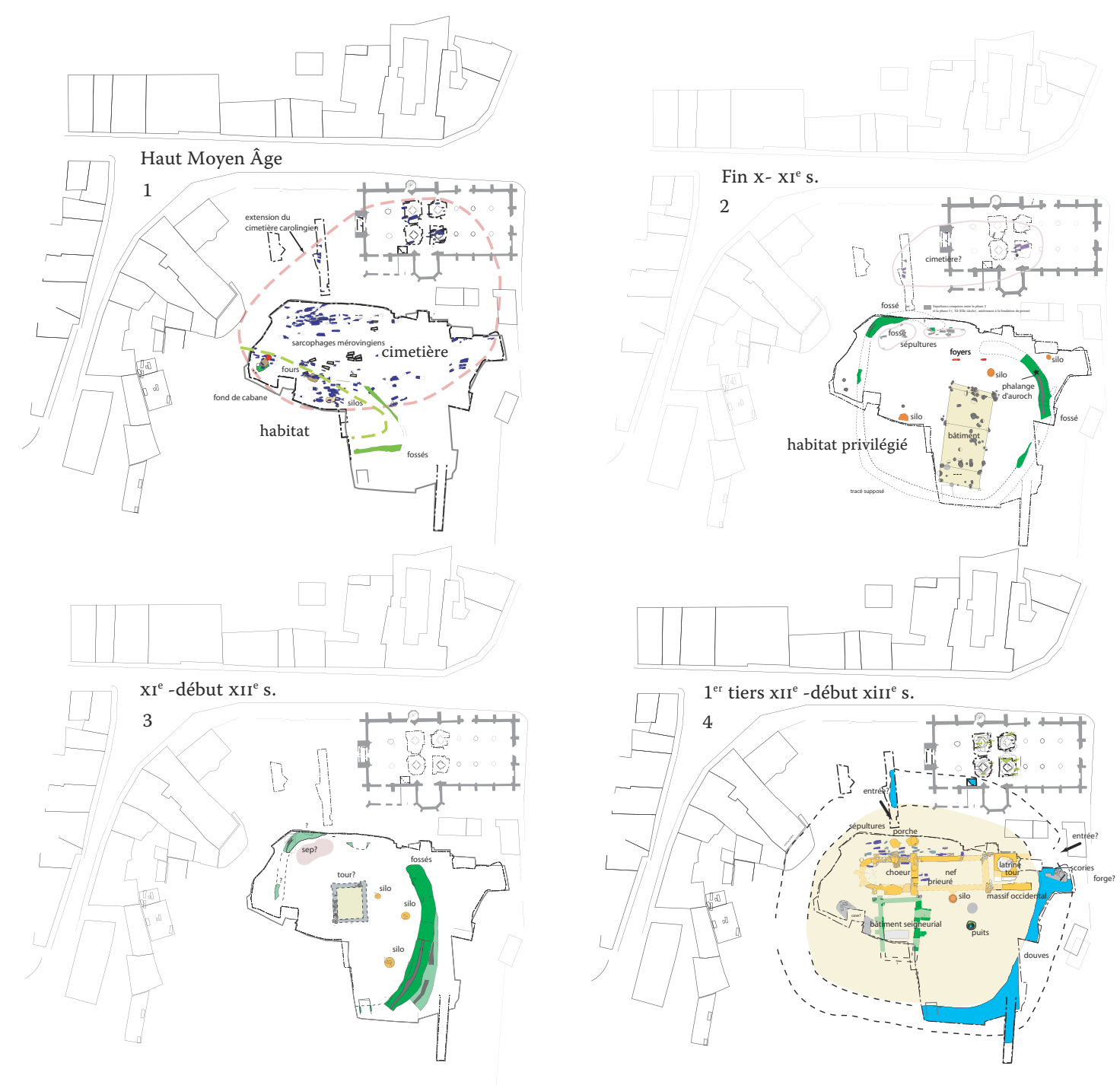

Fin $\mathrm{X}-\mathrm{XI}^{\mathrm{e}} \mathrm{s}$
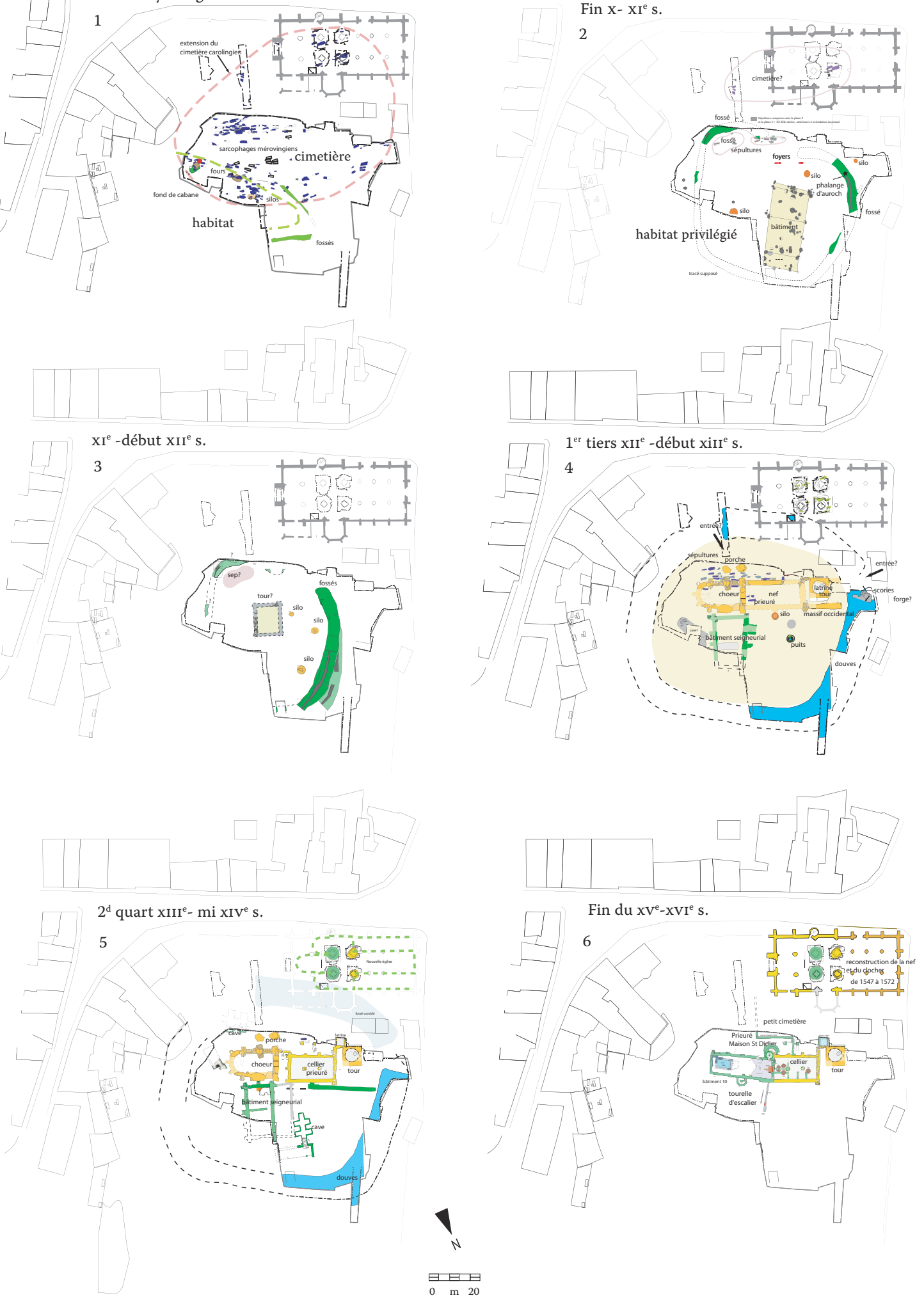

$2^{\mathrm{d}}$ quart $\mathrm{xIII}^{\mathrm{e}}-\mathrm{mi} \mathrm{xIV}^{\mathrm{e}} \mathrm{s}$.

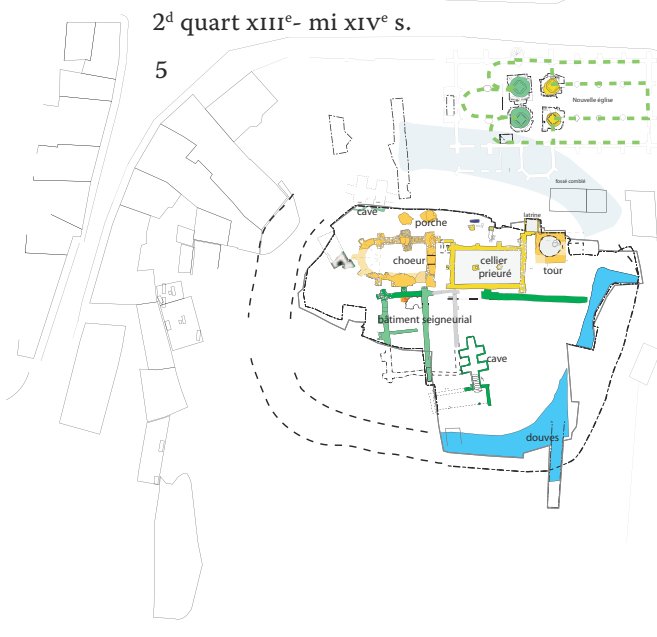


Le cimetière et l'habitat du haut Moyen Âge

À défaut de vestiges antiques, les premiers

éléments structurés sur la parcelle Saint-Didier appartiennent à la période mérovingienne.

L'implantation d'un espace funéraire au $\mathrm{VII}^{\mathrm{e}}$ siècle semble influencée par les facteurs conjugués:

l'existence d'un habitat adjacent, et la topographie qui confère au cimetière une situation finalement assez classique, sur une pente exposée au sud-est, à l'exact emplacement d'un carrefour de deux voies d'origine antique. L'habitat borde directement le cimetière, avec un empiètement réciproque entre les structures et les tombes.

L'origine du cimetière est associée à un groupe de sarcophages mérovingiens (VII ${ }^{\mathrm{e}}$ siècle) en plâtre, largement réemployés aux périodes suivantes. La variété des modes d'inhumation du viII ${ }^{\mathrm{e}} \mathrm{au}$ $\mathrm{XI}^{\mathrm{e}}$ siècle est frappante et le cimetière se développe de façon importante durant la période carolingienne (Abadie, 2014) du fait de l'extension de l'habitat à cette période (nouvel habitat de la Confiserie). Bien que le cœur de l'ensemble funéraire ait été entièrement observé, aucune trace d'édifice cultuel antérieur au XII ${ }^{\mathrm{e}}$ siècle n'a été notée et il reste peu d'espaces non explorés suffisants pour avoir accueilli un édifice de ce type: c'est une tout autre histoire qui se produit avec l'irruption d'un nouvel acteur, un habitat seigneurial.

\section{À l'origine de l'habitat seigneurial: une captation de l'espace funéraire}

On observe, à la charnière des $\mathrm{x}^{\mathrm{e}}-\mathrm{xI}^{\mathrm{e}}$ siècles l'implantation d'un vaste bâtiment sur poteaux plantés dont le plan nous est parvenu de façon cohérente malgré les nombreux recoupements postérieurs sur cette parcelle qui ont fait disparaitre une partie des trous de poteaux. Ce plan dessine une construction de $25 \times 11$ m orientée nord-sud. Il n'est pas installé au cœur du cimetière, mais légèrement décalé au nord: il est entouré d'un large fossé circulaire à profil en $V$ délimitant un espace d'environ $2000 \mathrm{~m}^{2}$ qui s'inscrit largement aux dépens du cimetière et recoupe systématiquement les sépultures antérieures. Dans cet espace interne, le bâtiment occupe la moitié ouest de la parcelle, les structures découvertes (silos, foyers) étant localisées préférentiellement entre le pignon sud du bâtiment et l'entrée de l'enclos. La découverte d'éléments de plâtre sur clayonnage, dans le remblai des trous de poteaux et du fossé adjacent, indique l'utilisation de ce matériau sur les murs du bâtiment.

L'étude archéozoologique lève toute ambiguïté concernant le caractère privilégié de cette occupation, avec l'association de plusieurs espèces chassées comme le lièvre, le cerf, le sanglier et surtout l'auroch. Encore chassé dans les Vosges par Charlemagne, l'auroch est par excellence un gibier de choix réservé au souverain et à ses proches (HennebicqueLe Jan, 1980). Il s'agit ici, avec l'exemplaire retrouvé sur le site des Halettes à Compiègne, de l'un des plus récents spécimens découvert avant la disparition totale de cette espèce de nos régions (Yvinec, 2014).
L'implantation de cet habitat dans le cimetière est donc le fait d'une élite locale, qui bénéficie de l'entourage du souverain et de la haute aristocratie. Nous ne pouvons assurer qu'il s'agisse déjà de la famille Le Bel, issue de l'entourage des Montmorency dont les premières mentions remontent à 1094, ou bien si ceux-ci ont pris la suite d'une famille plus ancienne. L'enjeu de captation d'un espace funéraire est très intéressant, en l'absence constatée d'édifice religieux. À Serris, à la même période, un grand bâtiment correspondant également à un édifice seigneurial est entouré d'un double fossé enserrant lui aussi le cimetière, dans lequel on suppose un édifice cultuel. Sur cette question, le parallèle entre ces deux sites semble assez évident (Gentili, 2010). Lévolution ultérieure de cet habitat confirme par ailleurs son statut seigneurial. Le fossé d'enclos est recreusé et son tracé légèrement modifié: c'est bien visible en partie ouest avec deux états successifs qui aboutissent à des fossés plus profonds. Un nouveau bâtiment un peu mieux centré dans l'espace fossoyé remplace la construction sur poteaux plantés. Son plan est lacunaire, mais deux murs aux fondations formées de petits blocs de gypse sont en partie conservés indiquant la position de deux angles opposés et l'indice de contreforts. Il pourrait s'agir d'un bâtiment de $9 \times 10 \mathrm{~m}$ de côté. La largeur des murs est de $1 \mathrm{~m}$, ce qui limite la hauteur de l'édifice tout en autorisant des étages, indiquant peut-être une sorte de tour résidence. Aucune trace de poteaux plantés n'a été relevée, ce qui n'empêche pas forcément l'utilisation de techniques de type pans de bois et plâtre sur clayonnage dans son architecture sur une partie de son élévation. Le bâtiment est associé à un groupe de silos qui sont les seules structures existant dans leur périmètre. La chronologie fine de cet état est difficile à établir à partir des seules données à notre disposition, mais semble correspondre au courant du XI ${ }^{\mathrm{e}}$ siècle et au plus tard au début $\mathrm{du} \mathrm{XIII}^{\mathrm{e}}$ siècle. Les structures les mieux datées étant les silos à grains, de forts volumes.

$\mathrm{Au} \mathrm{XII}{ }^{\mathrm{e}}$ siècle, les textes documentent cette élite locale. Les seigneurs de Villiers-le-Bel, la famille Le Bel, prospèrent grâce à leur relation avec l'abbaye de Saint-Denis dont ils sont vassaux. Vers 1146, Matthieu Le Bel, dans un aveu-lige rendu à l'abbé Suger, reconnaît tenir de Saint-Denis, outre ses biens propres, «cent quarante-sept fiefs dénombrés dans soixante-huit villages du Pays de France, quatre-vingt-quinze vassaux dont trente-deux chevaliers doivent l'estage avec obligation de résidence à la cour de Matthieu à Villiers-le-Bel» (Guadagnin, 200o, p. 28), ce qui nous donne, en prenant en compte les serviteurs, un effectif assez important de personnes à loger.

\section{Au XII ${ }^{e}$ siècle, la cohabitation habitat seigneurial - prieuré}

La reconstruction du bâtiment seigneurial s'accompagne dans le deuxième quart du XII ${ }^{\mathrm{e}}$ siècle d'un doublement de la surface enclose. Un large 
fossé à fond plat, creusé jusqu'à une nappe phréatique alimentée par les sources nombreuses au pied du mont Griffart, délimite une plateforme plus vaste $\left(3800 \mathrm{~m}^{2}\right.$ environ en incluant la surface des fossés), mais dont le point central reste identique à celui de l'enceinte précédente. L'irruption du religieux, pour être tardive, n'en est pas moins spectaculaire. En effet, l'espace nouvellement créé est partagé en deux moitiés presque égales par une construction nouvelle, désormais est-ouest, qui barre l'ensemble: les bâtiments d'un prieuré, prolongés à l'est par une église. L'ensemble, imposant, mesure 44. $10 \mathrm{~m}$. On peut restituer un édifice en trois parties : à l'ouest, une sorte de tour carrée à vocation résidentielle surmonte une vaste latrine, voûtée au centre, une nef prolongée d'un chœur voûté à contreforts massifs terminé en abside, au sud, l'accès étant matérialisé par un porche vraisemblablement lié à l'usage paroissial du lieu. Ces évolutions sont documentées par les textes, ce qui aide à leur datation, et s'inscrivent dans un phénomène plus large de création de prieurés associés à des édifices seigneuriaux (Racinet, 2012).

D’après l'abbé Lebeuf, Radulph Le Bel construit l'église de Villiers-le-Bel, qu'il remet à Étienne de Senlis, évêque de Paris, lequel la transmet en 1124 à l'abbaye de Saint-Victor de Paris (créée en 1113). En 1130, cinq religieux sont à demeure au prieurécure de Villiers-le-Bel, dont le nombre s'élève ensuite à huit entre 116o et 1169: Robert, prieur ; Hugues, Pierre, Grumbert, Turold, prêtres ; Simon, Hugues et Thibaud, diacres. En 1218, l'abbaye de Saint-Victor est autorisée à installer quatre chanoines si les revenus sont suffisants. Au sud du prieuré, un nouveau bâtiment seigneurial est construit, la nouvelle église mordant sur l'ancien édifice. Les restes de deux édifices superposés de dimensions assez proches, l'un du XII ${ }^{\mathrm{e}}$ et l'autre du XIII ${ }^{\mathrm{e}}$ siècle, d'orientation nord-sud, ont été découverts. L'état le plus ancien n'existe que sous la forme de lambeaux de tranchée de récupération située sous les murs du second édifice, lui aussi en partie récupéré. Les deux états de construction ont en commun un même matériau de fondation: blocs de gypse et grès liés au plâtre. Le second état présente des murs pourvus de gros contreforts indiquant une hauteur importante. Il mesure $20 \mathrm{~m}$ de long pour $9 \mathrm{~m}$ de large. Les restes de tuiles à glaçures jaunes, vertes et brun-rouge indiquent une toiture polychrome. L'espace interne est divisé en deux pièces égales par une cloison qui ménage une circulation le long du mur ouest.

\section{Au XIII ${ }^{\mathrm{e}}$ siècle, une nouvelle église}

L'intégration du prieuré au sein d'une nouvelle enceinte va poser des problèmes face à la rapide augmentation de la population durant cette période faste d'un point de vue démographique. La création du prieuré et la construction de l'église romane achèvent de saturer l'espace, malgré une extension de la surface enclose commune à l'hôtel et au prieuré. Les paroissiens sont trop nombreux pour l'église du prieuré; par ailleurs, la réduction de l'espace dédié aux inhumations va conduire à l'utilisation d'un autre cimetière (créé au XIII ${ }^{\mathrm{e}} \mathrm{ou}$ déjà existant?) qui sera dénommé ultérieurement le Grand cimetière (Guadagnin, 1981), l'ancien cimetière étant envahi par le bâti seigneurial et ecclésiastique. La création d'une nouvelle église, actée par un document de 1204, dont le programme architectural ambitieux nécessite de la place, va donc se faire par le lotissement d'une nouvelle parcelle au détriment du fossé d'enceinte comblé dans sa partie méridionale.

Malgré un rééquilibrage au $\mathrm{XIV}^{\mathrm{e}}$ siècle qui voit l'agrandissement de l'hôtel seigneurial, probablement couvert en ardoises, l'évolution va se faire au détriment du «château » et en faveur du prieuré dont le bâtiment principal est reconstruit avec, à sa base, un vaste cellier voûté.

\section{La fin de l'habitat seigneurial}

La messe semble dite dès le deuxième tiers du $\mathrm{XIV}^{\mathrm{e}}$ siècle lorsque l'hôtel cesse d'être la résidence du seigneur de Villiers désormais installé à l'IsleAdam. En 1464, l'hôtel seigneurial est mentionné comme «grand masure appelée la motte»

(Renaux, 1981, p. 73) tandis qu'à l'inverse, le prieuré héberge, à la Toussaint 1465, deux hôtes de marque: Louis XI et le comte de Charolais, futur Charles le Téméraire, à l'occasion d'une brève et houleuse entrevue.

À la fin du Xv ${ }^{\mathrm{e}}$ siècle (1499), le prieuré est agrandi après destruction complète de l'ancien hôtel seigneurial, et annexe l'ensemble de la parcelle dont les fossés sont comblés. La construction de ce nouvel édifice en plâtre et moellons est bien documentée par les comptes de fabrique ( ibid.) et présentait encore une partie de ses élévations à la fouille. À la même époque commencent des travaux sur l'église qui aboutissent au réhaussement de la nef et à la reconstruction du clocher : ils vont conférer à l'église de Villiers-le-Bel une envergure impressionnante et la silhouette de cathédrale qu'on lui connaît encore aujourd'hui (Foussard, 2008, p. 289-293). Malgré la crise des guerres de Religion, le prieuré-cure de Villiers-le-bel reste encore au XVII ${ }^{\mathrm{e}}$ siècle un ensemble prospère, outil de reconquête du catholicisme dans une localité à forte présence huguenote, dont témoignent les mémoires de Gourreau de la Proustière (Buffévent, 1990). Les lots de céramique (assiettes aux armoiries du prieur et verreries façon vénitienne) découverts dans ses latrines témoignent du statut du prieur, personnage clé de la localité.

Le souvenir de l'ancienne résidence seigneuriale s'estompe à tel point que les documents qui s'y réfèrent seront pendant longtemps attribués à la ferme seigneuriale située en partie aval du village. 
Comme on peut le voir dans l'exemple de Villiers-le-Bel, les fouilles préventives au cœur des villages actuels sont riches d'enseignements concernant la question complexe de formation du village médiéval et de l'évolution de ses composantes sociales. Ici, elles nous permettent de préciser les conditions d'émergence vers l'an mil d'un habitat élitaire dans le cimetière d'une villa carolingienne déjà importante à la fin du viII ${ }^{\mathrm{e}}$ siècle, et son évolution architecturale, menant d'un bâtiment sur poteaux au grand hôtel seigneurial des Villiers.

$\mathrm{Au} \mathrm{XII}{ }^{\mathrm{e}}$ siècle, la fondation d'un prieuré apporte une composante religieuse absente jusqu'alors, en faisant, par son importance jeu égal avec l'habitat seigneurial qui partage la même enceinte fossoyée. Cet espace est devenu vite trop étroit, transgressé par la fondation d'un nouveau sanctuaire au début du XIII ${ }^{\mathrm{e}}$ siècle. Ce processus aboutit, à la fin du Moyen Âge, à une situation inverse à celle de son commencement: seule subsiste sur la parcelle une église paroissiale imposante flanquée d'un prieuré prospère. Probablement absente au départ, la fonction religieuse du lieu l'a emporté au détriment des fonctions funéraires (à l'exception des sépultures ad sanctos à l'intérieur et en bordure immédiate de l'église) et seigneuriales occupant désormais des espaces excentrés du village.

Dans le cas de Villiers-le-Bel, les données de fouilles entrent en contradiction avec les premières interprétations privilégiant l'origine antique et le sanctuaire comme moteur de l'ancrage villageois. Elles apportent finalement un visage plus nuancé à cette question: ensembles funéraires, habitat des élites et établissement religieux jouant successivement leur partition, mais pas dans l'ordre imaginé au départ. Il convient de ne pas généraliser ces observations en modèle, la présence de sanctuaires du haut Moyen âge étant attestée dans de nombreux cas par les textes et l'archéologie, mais de montrer la variété des cas de figure qui nécessite, pour faire progresser cette question épineuse du village, la multiplication des études de cas de ce type.

Il ne faut pas se cacher la difficulté de l'entreprise: cette recherche est plus complexe et plus ingrate à mettre en œuvre que dans le cas d'opérations situées dans les champs. Elle porte généralement sur de petites parcelles et nécessite une politique volontariste de prescription par un suivi opiniâtre des permis de construire et la multiplication de petites opérations souvent contiguës, tirant partie des diagnostics comme des fouilles. La baisse des effectifs dans les services prescripteurs, les choix économiques privilégiant les diagnostics «rentables», la rupture de la chaîne opératoire entre diagnostics et fouilles, et la multiplication des opérateurs dans le cadre d'appels d'offre sont autant de risques et de freins à cette archéologie pourtant indispensable si l'on veut prendre en compte le phénomène complexe de formation et d'évolution des villages.

\section{Références bibliographiques}

ABADIE I., 2014, Étude de l'ensemble funéraire de Villiers-le-Bel-Saint-Didier, in Gentili F. (DIR.), 2014, p. 478-540.

Buffévent B. DE, 1990, Mémoires de Philippe Gourreau de La Proustière, Paris, Fédération des sociétés historiques.

Foussard D., Huet C., Lours M. (DIR.), 2008, Églises $d u$ Val-d'Oise, Pays de France, Vallée de Montmorency. Dix siècles d'art sacré aux portes de Paris, Gonesse, Société d'histoire et d'archéologie de Gonesse et du Pays de France, 308 p.

Gentili F (DIR.), 2008a, Villiers-le-Bel. La Confiserie, 72 rue Gambetta, Rapport final d'opération, Inrap CIF, Saint-Denis, $251 \mathrm{p}$.

Gentili F (DIR.), 2008b, Villiers-le-Bel, église SaintDidier, Rapport final d'opération de fouille préventive, Inrap CIF, Pantin, 12op.

Gentili F., 2010, Lorganisation spatiale des habitats ruraux du haut Moyen Âge: l'apport des grandes fouilles préventives. Deux exemples franciliens: Serris «Les Ruelles »(Seine-et-Marne) et Villiersle-Sec (Val-d'Oise), in Chapelot J. (DIR.), Trente ans d'archéologie médiévale en France. Un bilan pour un avenir, Actes du IX congrès international de la Société d'archéologie médiévale (Vincennes, 16-18 juin 2006), Caen, Publications du Crahm, p. 119-131.

Gentili F., 2011, «Une archéologie du village», Dossiers d'archéologie, 344, p. 32-37.

Gentili F., 2012, Prieuré et habitat seigneurial au coeur du village de Villiers-le-Bel: premières interprétations à l'issue des fouilles 2004-2012, Actes du premier colloque de Bellecroix (Chagny), 15-16 octobre 2011, Chagny, Centre de castellologie de Bourgogne.

Gentili F. (DIR.), 2014, Villiers-le-Bel, Rapport de fouilles archéologiques, Inrap CIF, Val-d'Oise, $782 \mathrm{p}$.

GUADAGNIN R., 1981, «Les quatre cimetières de Villiers-le-Bel », Bulletin de la JPGF, p. 10-11.

GuAdagnin R., 1982: L'Origine du village en Pays de France, Thèse de doctorat, École des hautes études en sciences sociales, 1982 .

Guadagnin R., 200o, Fosses-Vallée de l'Ysieux, mille ans de production céramique en Île-de-France, vol. I, Les données archéologiques et historiques, Caen, Publications du Crahm, 20oo, p. 23-71.

HenNebICQUe-Le Jan R., 1980, «Espaces sauvages et chasses royales dans le nord de la Francie, VII ${ }^{\mathrm{e}-}$ $\mathrm{IX}^{\mathrm{e}}$ siècles ", in Le paysage rural: réalité e représentations, Actes du $X^{e}$ congrès des historiens médiévistes de l'enseignement supérieur public, Lille, 18-19 mai 1979, Revue du Nord, LXII, n² 244, p. 35-57.

RACINET P., 2012, Du voisinage à l'insertion: étude des relations entre prieuré et château dans la moitié nord de la France (XI ${ }^{e}$-XIII ${ }^{e}$ siècle), Actes du premier colloque de Bellecroix (Chagny), 15-16 octobre 2011, Chagny, Centre de castellologie de Bourgogne.

Renaux D., 1981, Villiers-le-Bel:1428-1499, Mémoire de maîtrise d'histoire, École des hautes études en sciences sociales, $153 \mathrm{p}$.

WARMÉ N., Gentili F, 2012, Villiers-le-Bel, 5, rue Louise Michel, Rapport de diagnostic archéologique, Inrap CIF, 68 p.

YVINEC J.-H., 2014, «Étude archéozoologique des contextes médiévaux et modernes de Villiers-leBel », in Gentill F. (DIR.), 2014, p. 414-432. 